\title{
MCQ Exams Correction in an Offline Network Using XML
}

\author{
Jehad Al-Sadi \\ Arab Open University \\ Jehad@al-sadi.com
}

\author{
Daed Al-Halabi \\ What is Next? \\ Daed_halabi@yahoo.com
}

\author{
Hasan Al-Halabi \\ What is Next? \\ Hasanhalabi@gmail.com
}

\begin{abstract}
One of vital subject in education facility is student assesment. A common way used to compute there work is making exams. Generaly class sizes tend to expand in some socities. As a result there is a trend to give a quick accurate evaluation which is become more demand. A computerized questions make the process of taking an exam easier and somther. This caused the move towards the use of multiple-choice questions (MCQ). The rapid progress of using XML (Extensible Markup Language) for large amount of structured data, due to its ability of saving time and manipulate data makes it suitable for MCQ exam environment. Moreover, XML manipulates and deals with networks suffering from failure occurrences. The main contribution of this paper is to present an efficient method of transfer data related to online questions between the server and clients stations without being affected if the connection fails during taking the exam. An analytical study of the efficiency of module is also presented.
\end{abstract}

\section{INTRODUCTION}

One of the main topics in the educational process is the quality and the value of students' work, such as information correctness, fair assessment methodology that illustrates indepth thinking and creativity. $[1,3]$

Assessment is not easy for both of lecturers and students. In general, student assessment through taking exams is the main way to measure students' performance in a given course. $[1,2]$

Since there is a dramatic growth in students' numbers and in the sizes of some classes, a quick assessment is a necessity and it is an important issue in the university environment [2]. It helps them to improve their thinking skills and focus on the essential vital purposes for what they learn rather than distract their attention towards different insignificant objectives and outcomes. [1]

Logically, the acceleration in financial and political pressure issues increased by raising students' numbers and course diversity payments strategies. To achieve these objectives and increase consistency between social requirements and criteria, automated assessment is in place. $[3,4]$

Therefore; MCQs type of exams is suitable for this situation. MCQ exams are considered a fast learning measuring technique. They help students to progress as far and quickly as possible, and document that progress and achievement [4].

MCQ is a summative assessment used to give measurable indication for students' achievements and intellectual skills. MCQ test has recently become an essential part of the assessment for different courses, in particular, large classes where grading consumes more time and resources. [4, 5]

Naturally, the number of participants taking a particular test has become very large and/or the number of questions is large. This is to be done through the application by comparing student answers with the correct answers provided by the examiner. This is computerized to release the results of the examination as quickly as possible.

The regular exam dialogue for a student using an online network involves answering questions or exam forms from a server. First, the server needs to authenticate the student and retrieve the correct exam for him. After the student completes the exam, the server must ensure the student's answers are saved and score the exam using the answer key that was provided by the teacher.

Therefore, many network transactions are required between client and server within the exam period to accomplish the exam workflow. If the network faces periodic disconnection problems, the transactions of data transfers will lead to data losses and delay. At the end, the server may not have a correct version of the student's answer and the student will not have his full time in the exam because of the constant delay.

This paper is organized in the following manner: Section 2 describes the existing cases in real current situations. Section 3 distinguishes the pros and cons between online and offline application. Section 4, describes system requirements besides the suitability of XML for MCQ exams and advantages and disadvantages of using MCQ exams. Section 5 describes in detail how a grade is obtained automatically from an MCQ exam, it's workflow, it's correction procedure and the security issues needed. Section 6 shows the analysis and complexity of the suggest solution. Finally section 7 presents conclusion.

\section{BACKGROUND}

Existing MCQ systems are usually uses in online local network environments (e.g. Blackboard, WebCT, and TopClass). In general, those systems use a static exam 
questions, or generate different forms of exam. On the other hand, an enhancement and development can produce different exam questions to make an exam using XML [6].

Those systems and others are based on a client/server structure relying on the assumption that the network is accessible most of the time. In reality, a failure in a network is always a possibility in low-budget institutions. A network failure can occur due to several reasons as its infrastructure errors that occur from designing to implementation stages; such as the purpose of creation, how many clients will served, its functionality, communication lines or different points of failure, etc. $[7,8]$

That implies wither an online network is available in a percent of $\mathrm{k}$, where $\mathrm{k}<100 \%$; $\mathrm{k}$ represents the connectivity of the network. On the other hand, offline indicates that network functions are down for lots of problems. In other words, a network is not accessible in (100-k) \%. [8] Our concern focuses on the situation where a client is running an exam where the network may be disconnected from the server. The target is to keep MCQ exam running smoothly in a network that lost the connection to its server for a certain period.

To be able to collect these information together, when having offline network while having to apply MCQ test; yields to the need of using XML-utility as a suitable solution in such situation. XML flexibility of carrying and interchanging data when a direct connection between client and server most of the time is not available make it a proper solution tool to use.

\section{COMPARING ON-LINE AND OFFLINE APPLICATION}

In simple comparison between the offline and online web pages solutions, the online solutions will be better in many cases, for example:

- The data transmitted in the online solution is much less than the data transmitted in the offline solution.

- In the online solution, the server can automatically find the absent students in the exam.

- In the online solution, the server can automatically find the duplicated student IDs.

However, when the network is unreliable with many failures, or in case of homework when the student's presence is not mandatory, the offline solution will be much better than the online, as it is beneficial for the student.

\section{MCQ IN AN OFFLINE NETWORK USING XML}

\section{Preparations}

Here is a closer look at solving this case, firstly we have to check that hardware, software, and human factors are available.

Hardware workstations consist of a personal computer, a requirement for each student. For software, the suggested solution uses XML with suitable programming language related to .Net Technology or Java technology. Human factors include course coordinator, students, and lab supervisor.

A course coordinator is mainly responsible for putting exam questions. Every registered student attends the exam; his answers and grade saved in his record.

XML Offline MCQ system as an integrated system that has two components

1. Server component: main requirement is XML MCQ questions pool, students' lists for each exam and other proper information.

2. Client Component: a suitable GUI (Graphical User Interface) to display MCQ exam with a functionality to save student answer, and an application that can communicate with the server.

\section{A. MCQ - Its Demands, Objectives, and Design Issues}

MCQ exam is considered as a self-learning tool that concentrates on the understanding of subject principles. It is used to give valuable accurate measurement and assessment strategies to achieve good monitoring, update metrics to meet students' needs and encourage their effort and work. [4, 5]

Advantages of having MCQ exam [5, 9]:

1. Effective and easy way for evaluation in an environment of large number of students.

2. Automatic scoring.

3. Gives well documented feedback on students' learning outcomes.

4. Questions stored in questions poll may be used for several times.

Disadvantage of having MCQ exam [5, 9]:

1. Gathering, assembling, and building good meaningful items are time consuming.

2. Tests knowledge and memorization.

3. Students' capabilities of writing skills and analysis skills cannot be properly tested.

4. Surface approach for guessing may be persuaded.

\section{B. XML Use}

$\mathrm{XML}$ is a structured document layout used to exchange data in a flexible way. XML is a generalpurpose specification for creating custom markup languages. [10]

An XML schema describes the hierarchy structure of an XML document. An XML schema provides a view of the document type at a relatively high level of abstraction. Additionally, it allows machines to carry out rules made by people. [10]

XML offers its users many advantages, including [6, $10]$ 
- Simplicity

- Extensibility

- Interoperability

- Openness

The reasons for choosing XML that it is:

1. A well known data representation format (structured and standard)

2. Supported in most programming languages.

3. Easy to be sent through the networks.

4. Ability to encrypt and decrypt is easily.

XML databases store data originally in its structured, hierarchical form. Queries can be resolved much faster because there is no need to map the XML data, since it has a tree structure map directly to tables. This preserves the hierarchy of the data and increases performance. $[6,10]$

The characteristics of data using XML $[6,10]$

1. Self-describing data

2. Capability to integrate all traditional databases and formats (data type - text, numbers, or multimedia objects and sounds [using text representation i.e. Base64]), to active formats like Java applets or ActiveX components.

3. Ability to alternate data presentation (no reprogramming required).

4. One-server view of distributed data (XML documents can consist of data from many different databases distributed over multiple servers).

5. Open and extensible

An example of used XML for MCQ simple structure is shown in figure 1 from line 2 to 3 . Describe MCQ in structured hierarchy XML tree that a MCQquestion consists of question stem, question answer key, grade weight and questions options.

1. $<$ ExamForm ID="44" CourseNo= "8982345"

Semester $=$ "First" ExamLevel= " 1 " $>$

2. $<$ Question ID="7" AnswerKey ="3" GradeWeight $=" 2 ">$

$<$ Stem $>$ what is the capital of Jordan? $</$ Stem $>$

$<$ Distractor $>$ Oman $</$ Distractor $>$

$<$ Distractor $>$ Dubai $</$ Distractor $>$

$<$ Distractor $>$ Amman $<$ /Distractor $>$

$<$ Distractor $>$ Jerusalem $<$ Distractor $>$

3. $</$ Question $>$

4. $</$ ExamForm $>$

Fig. 1. An XML snapshot of ExamForm file.

\section{APPLICATION DESIGN ISSUES AND ITS ANALYSIS}

\section{A. The Workflow Model for a MCQ Exam}

In the exam, a student has to set down to apply the exam for a limited time to complete it. The exam applied on a network has numerous time of fail (i.e. get down). In like this situation, it produces a problem that appears with data consistency and its integrity.

An automatic correction for MCQ environment has to guarantee data consistency and its integrity for both students' marks saved in their records correctly, and teachers (they have to see it as trustworthy). A copy of every student's answer of an exam must be saved after it is been automatically corrected (the system should provide assurance to keep a copy of it), obtained grade, the assessment of correction way, etc.

In this design methodology, the integrity of the data insured by the XML itself, because it is structure data and it can be easily integrated with any other database management system. However, the consistency is a deferent manner; the consistency of the application depends on the network failure after the student performs the exam. (Data will take time until it becomes consistence).

Thus, an offline solution will be a very good solution. In some places, fixing the network problem and upgrading its infrastructure will cost the educational facility much more than the existing budget. Thus, an offline network solution for the exams will be the right choice.

The basic idea of this solution is to transfer questions of the exam using XML in one packet from the server. Then, make the students answer these questions and store the answers in an encrypted XML file, in the client workstation without any communication with the server. After the student finishes answering and closes the exam. The workstation will send the answers back to the server without affecting students' time.

Success of this infrastructure depends on the design of the client application, which will keep trying to guarantee receiving the XML file of the exam from the server before the exam time starts. This also applies on sending the answers back.

The basic scenario for this application is as the following:

1. The exam administrator has to make sure that each workstation client in the exam start getting the exams of that day from the server. In case the network failure has extended to the time of the exam, the administrator shall have the ability to install the exam into the client machines using offline solution like Flash Memory or CDs.

2. Each client will get the exam name, course code, start time, end time, questions, and student who must apply for the exam. The student information will include name, ID number, and authentication information.

3. This amount of data is much larger than the amount 
transmitted in the usual online application, but on the other hand these data will be transmitted before the exam starts and will keep the student away from the network failures.

4. When the exam time is due, the student will fill in his ID and authenticate himself/herself. The client application on the workstation will verify the information with the information stored in it about the exam. When the information is verified, the application will open the exam, and the student will start answering. Otherwise, the application will keep the exam close.

5. After the student finishes answering the questions or the exam time is over, the application will close the exam and prevent the student from answering.

6. Here are two approaches that may be used by the educational institute. The first is to let the client application correct the exam directly and give the grade to the student, or send back the answers to the server and make the correction on the server itself.

7. Whatever the approach the educational institute chooses to use to correct the exam. The client application will save the exam answers and student information in an encrypted XML file and send it back to the server when the server is reachable.

Figure 2 shows the flowchart for automatic correcting of MCQ exam process. The flowchart shows sequence path of exam procedure.

\section{B. Security}

In MCQ exam, it is important to transmit secure information XML-file with suitable format over the network. Variant security issues arise including exam date, user authorization, encryption/ decryption XML files, and exam time.

The automatic correction MCQ exam procedures constraints are:

1. User authorization: the system has to check if the user-ID registered in the course under certain section.

2. Authentication method determined and provided by the university/institute system.

3. It is not allowed for a student who has a username with authentication to take the exam except in its due date.

4. The automatic correction MCQ system has to ensure flexible policy in attending any exam sections for students who have accepted circumstances.

5. Encryption/decryption methodology used in sending exam questions and student answers.

6. Exam corrections shall be done after all exams have been completed at the end of the day or as soon as the student finishes answering it.

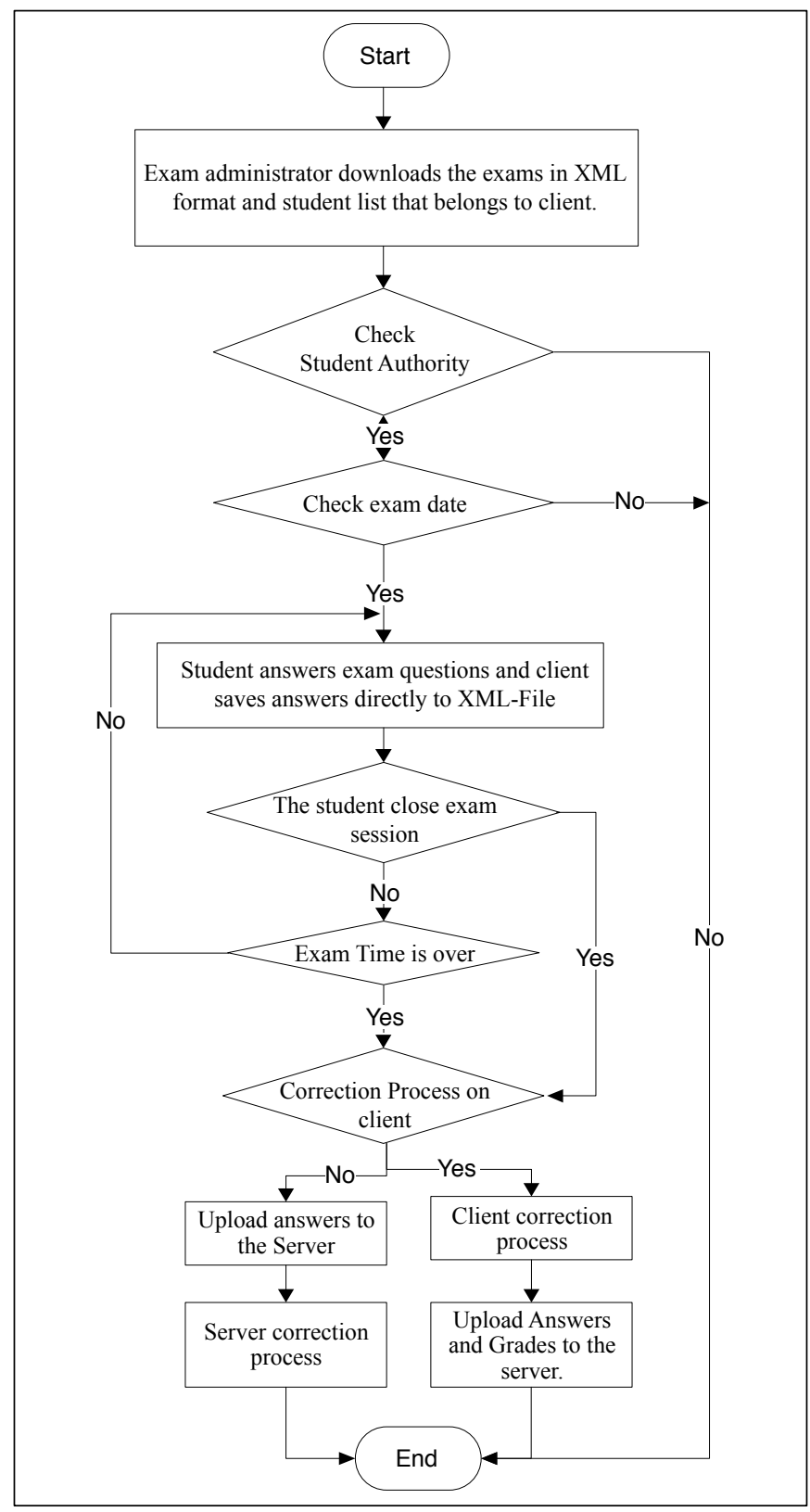

Fig. 2. the flowchart for automatic correcting for MCQ exam process

Although, there are many shortages that need special procedure to make sure that it will not brake the exam security including:

1. Human inference at workstation level, this can occur at any time before or after exam time while the exam still saved at the workstation, before it is transferred back to the server.

2. Inconsistency of automatic correction if it is conducted at workstation level. The correction schema may vary between different workstations unless there is an assurance of consistency among all workstations.

\section{ALGORITHM ANALYSIS}

In this section we present an analytical module to study 
our methodology of conduction of MCQ exams in terms of speedup, big-o and other metrics.

\section{A. Algorithm Preparation}

Performance metric analysis as speedup and other tools used as helpful tools to indicate and prove validation of the presented concept. [11, 12]

The client/ server interaction with each other is mainly our concern, in addition to the exam correction process.

However, an institution should be able to take care of changing conditions with capabilities such as maximizing numbers of students who attend the exam class, new technologies, defining transitions and implementation paths, etc.

Measuring the complexity and performance of any algorithm is the main factor to prove its applicability. Therefore, it is an important procedure to conduct such a study.

\section{B. Complexity measure}

By default, a client/server network is a distributed system. Based on this fact, finding the computing complexity of algorithm performance is not an easy task. Many factors arise from network issues (hardware and software). To compute the complexity of XML MCQ exam on a network, the exam procedure is divided into three basic stages.

1. Server has to supply clients with required information. (Exam information, students list, etc).

2. A student takes the exam on a client, save exam answer on a client workstation, and then the correction stage for the exam is performed.

3. Transfer students' answers to the server to keep it in student record.

Focusing on the first stage, the server broadcast information organized in files to k-clients. The main central point is the size of data that need to be moved from server to k-clients on connection lines between them.

Also this is applied to stage 3; there are transitions of information organized in files from k-clients transferred to server.

Moreover, keep in mind the network suffers from many assumed failures. To compute the worthiness or efficiency of the exam process applied on this framework yield the need of a well study on time, memory, and number of clients. Beside there are sets of interrelated factors taken into consideration as communication, synchronization, fault tolerance, etc.

Time is the most basic element that gives an excellent indicator in design and implementation of an application. It is affected by the design of the network. [11, 12] A scientific approach is used to predicate the needed time from start to end the process. We may define it as the total amount of time of transmitting the required files (stage 1) adding to it the correction step (stage 2). In addition, the total amount of time spent on the transmission of the students' answer files is to be kept it in his/her records at the server side (stage 3 ).

The amount of failure time of connection from server to a client or vice versa is also considered.

The efficiency measurement depends on big-O analysis, as follows:

$F(O(X))=$ Total time that occurred before correction procedure (step 1) + correction stage ( step 2) + Total time occurred after correction done (step 3 )

The following notations will be used in the next subsections to measure the performance analysis:

$\mathrm{K}$ is number of clients in client/server network.

$\mathrm{N}$ total numbers of students take MCQ exam. $\mathrm{m}$ is the number of questions in an exam. $\mathrm{v}$ is the number of amount transmit data in files from server to a client.

$N O_{T f_{\text {server }}}$ is number of failing tries to get connection to reach required information from server to client. (Step 1)

$N O_{T f_{\text {client }}}$ is number of failing tries to get connection to reach required information from client to server. (Step 3)

\section{Correction Algorithm}

Either correction process is compute locally on client or on server. The input files required for this process is ExamForm and StudentAnswer files, both files of type XML. Figures 1 and 3 illustrate samples of information the module contains. Figure 1 contains essential fields for an exam saved in (ExamForm. $\mathrm{xml}$ file). These fields are form ID, course number, exam semester and exam level. In addition to MCQquestions which consist of information about the questions including the awarded grade for every question.

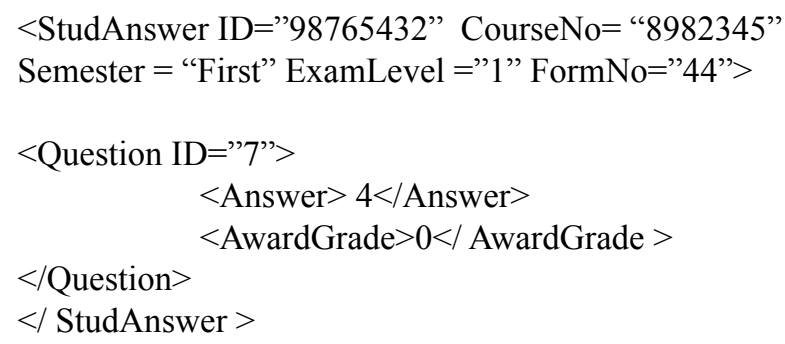

Fig. 3. An XML snapshot of StudentAnswer file.

Figure 3 contains essential fields belongs to student answer file. They are student ID, course number, exam semester, exam level, and form number. In addition to the corrected MCQ-questions, and student achieved grade. 
The algorithm of correction MCQ exams is described below.

Description: The correction algorithm.

Input: ExamForm.xml.and StudAnswer.xml Output: StudGrade.xml.

Method:

1) Initialization:

a) Open the student answers XML file named (StudAnswer.xml).

b) Get StudNo from the StudAnswer.XML and put it in the variable StudNo

c) $\mathrm{Sum}=0$

d) Open the exam form file named (ExamForm. $\mathrm{xml}$ )

e) Get FormNo and put it in the variable FormNo

2) Algorithm, Process: Correction

Check form number (FormNo) in both input files if they are the same otherwise print an error message.

a) Write in the output file (StudGrade.xml) StudNo and FormNo.

b) While not end of file (EOF) for both files StudAnswer.xml, and ExamForm.xml.

\{

If StudAnswer.Answer $==$ ExamForm. AnswerKey Then

AwardGrade= ExamForm. GradeWeight.

else

AwardGrade $=0$

Write in the output file, StudGrade.xml, the question ID and the AwardGrade for that question.

Sum $=$ Sum + Award Grade

\}

3) Write the Obtained Grade summation in the output file

a) Write sum in the output file (StudGrade. XML)

To analyze the correction algorithm for an exam has (m) questions; mainly, analyze the repetition statement (while at 2-b). This stage will pass on the whole StudAnswer.XML file for each student is $\mathrm{m}$ times. The correction algorithm will take $\mathrm{O}$ (m). Assuming that there are $\mathrm{N}$ students applying the exam, it will produce $\mathrm{O}(\mathrm{m} \times \mathrm{N})$ which yield to $\mathrm{O}(\mathrm{N})$. An observation on the correction process is its runtime function is linear.

\section{Files Transactions between Clients and Server}

Obviously, a transaction from server to client and vice versa is time consuming over a network. The need to know network performance is elementary. Therefore, Compute:

- Preparation step (information broadcast): A number of transmitted input required files (v) from server to each $\mathrm{k}$-clients $(\mathrm{v} \times \mathrm{k})$ (This step occur once for a certain exam), add to it number of try to get a connection from server to each client is the summation for fail tries for each client.

$N O_{T f_{\text {server }}}=\sum_{i=1}^{i=K} \sum_{\text {between server and a client }}{ }_{1}$ No Fail Tries to get connection

- Final stage (information collection): A number of transmit output required files $(\mathrm{N})$ from clients to server, add to it number of try to get a connection for each client to server is summation for number of fail tries from each client to server. .

$N O_{T f_{\text {client }}}=\sum_{i=1}^{K} \sum \begin{aligned} & \text { No Fail Tries to get connection } \\ & \text { between a client and server }\end{aligned}$

Collect all previous sub parts and substitute them in equation (1):

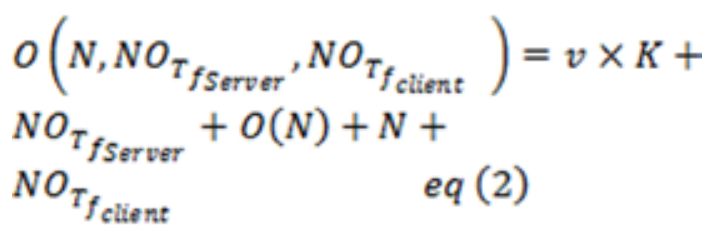

By eliminating constants $(\mathrm{v}, \mathrm{K})$ from the equation (2), the result is

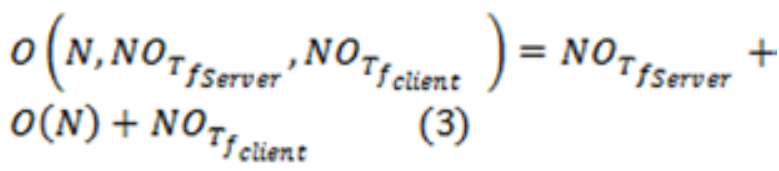

In the end, the Big-O for both corrections methods (On Client Or Server) are the same but the difference is that in the first method is the load of the calculation for total grade for $\mathrm{N}$-students exams, will be divided on more than one machine ( $\mathrm{K}$ machines which the facility have). On the other hand, the second method will put the correction load on one machine which is the server.

\section{E. Performance metric for the system}

In general, to compute the performance of suggested XML MCQ exam in offline network model, a comparison made with existing XML MCQ exam in on-line network model to solve the same problem.

The main property of online network is its reliability. Its time element reduced to be near zero using hardware/ software technologies with its basic operation send and receive. Generally, many metric exist study the performance of a system. Most 
common measurements are speedup, efficiency and a cost related to time element is used.

Define

$$
\begin{aligned}
& N O_{T f_{\text {server }}} \approx 0 \\
& N O_{T f_{\text {client }}} \approx 0
\end{aligned}
$$

Substitute them in equation (1):

$$
\begin{aligned}
& \mathrm{O}(\mathrm{N})=\mathrm{O}(\mathrm{N})+\text { Zero }+ \text { zero } \\
& \mathrm{O}(\mathrm{N})=\mathrm{O}(\mathrm{N})
\end{aligned}
$$

The problem solved in $\mathrm{O}(\mathrm{N})$ time on online network.

Speedup (S): defined as the ratio of a sequential algorithm to parallel algorithm. We are going compute the speed up as the ratio of the same algorithm run on online environment to run time on an offline algorithm.

$$
S=\frac{N}{N+N O_{T_{\text {Server }}}+N O_{T_{f_{\text {client }}}}}
$$

Efficiency: defined as the ratio of speed up to the number of processors (Clients).

$$
\begin{aligned}
& \mathrm{E}=\mathrm{S} / \# \text { clients } \\
& \mathrm{E}=\mathrm{O}(\mathrm{S}) / \mathrm{K}
\end{aligned}
$$

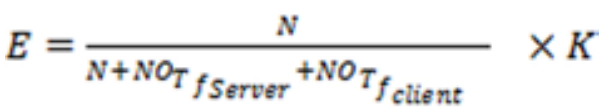

Cost evaluated as the result of the production of run time and number of processors used. That produces (in exam round):

$$
\text { Cost }=\left(N+N O_{\tau_{\text {fServer }}}+N O_{\tau_{f_{\text {client }}}}\right) \times K
$$

\section{CONCLUSION}

To enhance student information and knowledge therefore different types of questions are used, one of them is MCQ. MCQ basically deliver basic information and knowledge within a reasonable rate.

A highly political and society demand encourage the movement towards automatic correction for MCQ exam. XML technology used for an automatic correction for MCQ exam using under client-server environment that has several disconnection problems in its network infrastructure has been presented. The paper has presented two solutions for MCQ exam correction; either it is done on client workstations or on server. Obvious time spent to achieve data consistency in its database is mainly required overhead work done on communication lines. An analysis for their run time, big-O, speedup, efficiency and cost is evaluated to give good indicator on applying the suggested MCQ exam in offline network. The paper also presented the accurse and security factors of the new method by preventing lose of data while students conducting an exams at the present of connection failures.

\section{REFERENCES}

[1] The Community College of Baltimore County, 2008, Guide for Learning Outcomes Assessment \& Classroom Learning Assessment http://www.ccbcmd.edu/media/loa/loa_booklet.pdf

[2] Rust, c., Price M. and O’donovan, B. 2003. Improving Students' Learning by Developing Their Understanding of Assessment Criteria and Processes, Assessment \& Evaluation in Higher Education, Vol. 28, No. 2, (pp. $147-164$ )

[3] The University's approach to quality management, Learning and Teaching Enhancement Office, University of Bath, Viewed 5/3/2010, http://www.bath.ac.uk/learningandteaching/cop/qastatements/ qualitymanagement

[4] Dennis L., Mills .S., Smith P., Tucker A. and Exely K. , Automated Assessment for Large Groups, School of Computer Science, University of Nottingham , http://www.cs.nott.ac.uk/ smx/PGCHE/MCQ.html.

[5] Roberts T. S., 2006, The Use of Multiple Choice Tests for Formative and Summative Assessment, Proceedings of the 8th Austalian conference on Computing education, Volume 52, pp. 175 - 180

[6] Lister R. and Jerram P., 2001, Design for Web-Based On-Demand Multiple Choice Exams Using XML, Proceedings of the IEEE International Conference on Advanced Learning Techniques (ICALT'01), pp 383-384

[7] Aiken R. J., Boroumand J., and Wolff S., 2004, Network and Computing Research Infrastructure: BACK TO future, Communication of the ACM, Vol. 47, No. 1, Pages: 93 - 98

[8] Andrew S. Tanenbaum, 2003, Computer Networks, 4th edition, Prentice Hall

[9] Crocker L., Schmitt A., 1987, Improving Multiple-Choice Test Performance for Examinees with Different Levels of Test Anxiety, Journal of Experimental Education, Vol. 55.

[10] Silberschatz A., Korth H. F. and Sudarshan S., 2002 , Database system concept, 4th edition, Mc Graw Hill.

[11] Kumar V, Grama A., Gupta A. and Karypis G, 1994, An Introduction to Parallel Algorithms: design and analysis of algorithms, BenjaminCummings Publishing Company.

[12] Ghosh S, 2004, Algorithm Design for network Information Technology Systems, Springer-Verlag New York

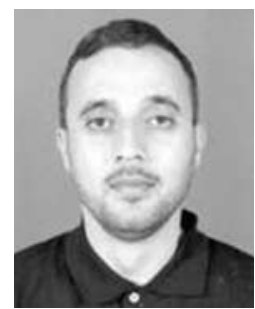

Jehad Al-Sadi received his B.Sc. degree in Computer Science from Tennessee State University, USA, in 1989, and M.Sc degree in Computer Science from Jackson State University, USA, in 1993. From graduation till 1996, he was a lecturer in the Department of Computing Science at the Al-Isra University, Jordan. He gained his Ph.D. in Computer Science from the University of Glasgow, U.K. 2002. He joined the Computer Science Department at Zarka Private University, Jordan from 2002 until 2004. He is currently an Associate Professor and the Chairman of IT and computing department at Arab Open University, Jordan. His research interests are: interconnection networks, optical networks, fault-tolerant routing, elearning, and learning management systems.

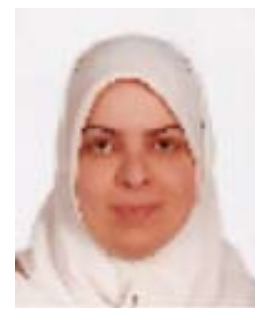

Daed Al-Halabi received the MSC degree in Computer Science from Jordan University, Jordan, in 2000. She is currently working in What Is Next? for technology solutions. She spent around 10 years working in high education institutions. Her research interest includes software development with a focus on Quality of Service.

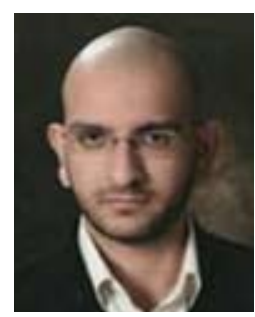

Hasan Al-Halabi received the B.Sc degree in IT from Balqa University, Jordan, in 2004. He is currently working in What Is Next? for technology solutions. He worked 5 years in various establishments related to IT (software and hardware) sectors. His interest areas are in business development, sales \& marketing, as well as general management areas. 